\title{
Retrospective evaluation of histopathological examinations in invasive ductal breast cancer of no special type: an analysis of 691 patients
}

\author{
Anna M. Badowska-Kozakiewicz ${ }^{1}$, Anna Liszcz ${ }^{2}$, Maria Sobol ${ }^{1}$, Janusz Patera ${ }^{3}$
}

\begin{abstract}
${ }^{1}$ Department of Human Biophysics and Physiology, Medical University of Warsaw, Warsaw, Poland

${ }^{2}$ Student Scientific Group of Cancer Cell Biology, Department of Human Biophysics and Physiology, Medical University of Warsaw, Warsaw, Poland

${ }^{3}$ Department of Pathomorphology, Military Institute of Medicine, Warsaw, Poland
\end{abstract}

Submitted: 24 April 2015

Accepted: 8 July 2015

Arch Med Sci 2017; 13, 6: 1408-1415

DOI: 10.5114 /aoms.2015.53964

Copyright @ 2016 Termedia \& Banach

\section{Abstract}

Introduction: Invasive ductal carcinoma (IDC) is the most common type of breast cancer in women and accounts for about $80 \%$ of all breast cancers.

Material and methods: The material consisted of histological preparations derived from 691 patients treated for IDC-NST.

Results: In our own study material, invasive ductal breast cancer of no special type accounted for more than $60 \%$ of cases, with the largest percentage of tumors being classified as G2 (53.96\%) and G3 (28.98\%). In terms of tumor size, the most common IDC-NST tumors were those of stage T1C (34.59\%) and T2 (35.31\%). The incidence of lymph node involvement was also assessed to reveal that no lymph node metastases were present in $45.44 \%$ of IDC-NST tumors. In the histopathological analysis of IDC-NST, significant statistical correlation was demonstrated between the presence of lymph node metastases and the histological malignancy grade (NO/G1-G3 $p=0.0103 ; \mathrm{N} 1 \mathrm{~A} / \mathrm{G} 1-\mathrm{G} 3 p=0.0498 ; \mathrm{N} 1 \mathrm{~B} / \mathrm{G} 1-\mathrm{G} 3 \quad p<0.001 ; \mathrm{N} 3 / \mathrm{G} 1-\mathrm{G} 3$ $p=0.0027$; N4/G1-G3 $p<0.001$ ), between the presence of lymph node metastases and the tumor size (N0/T1-T4 $p=0.00295$; N1B/T1-T4 $p<0.001$; N2/T1-T4 $p<0.001 ; \mathrm{N} 2 \mathrm{~A} / \mathrm{T} 1-\mathrm{T} 2 p<0.001 ; \mathrm{N} 4 / \mathrm{T} 1-\mathrm{T} 4 p<0.001 ; \mathrm{Nx} / \mathrm{T} 1-\mathrm{T} 4$ $p=0.0447)$, as well as between the histological malignancy grade and the tumor size (G1/T1-T4 $p<0.001$; G1/2/T1-T4 $p<0.001$; G2/3/T1-T4 $p<0.0267$ ). Conclusions: Own research demonstrated that the most common histological type of breast cancer is invasive ductal carcinoma of no special type (IDC-NST); statistically significant correlations were demonstrated in IDCNST patients between the lymph node involvement status and the histological malignancy grade or tumor size as well as between the histological malignancy grade and the tumor size.

Key words: invasive ductal carcinoma of no special type, histological grade, tumor size, lymph node status.

\section{Introduction}

Breast cancer is a heterogeneous disease, comprising multiple entities associated with distinctive histological and biological features, clinical presentations and behaviors, as well as responses to therapy [1-5]. Inva-

\author{
Corresponding author: \\ Anna M. Badowska- \\ Kozakiewicz \\ Department of Human \\ Biophysics and Physiology \\ Medical University of Warsaw \\ 61 Zwirki i Wigury St \\ 02-091 Warsaw, Poland \\ Phone/fax: +48 226287846 \\ E-mail: \\ abadowska@wum.edu.pl
}


sive ductal breast cancer is the most common type of breast cancer in women and accounts for about $80 \%$ of all breast cancers [6-8]. The terminology ductal is still being used for historical reasons and to date there is no evidence to suggest that these tumors arise from ductal epithelial cells [9-11]. The reason why it is sometimes referred to as "no special type" (NST) is that the cells under the microscope have no particular features that would classify them as a specific type of breast cancer $[10,11]$. This is basically an acknowledgment that it has been impossible to classify the carcinoma as a particular type, and consequently it has been placed in this holding area until further classification strategies become available [11]. It is also not the case that such lesions necessarily derive from, or form, ductal structures; thus the term "no special type" is preferred by some pathologists over the name ductal [12]. Macroscopically, invasive ductal carcinoma NST occurs most often in the form of a hard tumor visible in the cross-section with an irregular contour $[9,11,12]$. Focal necrosis and an associated chronic inflammatory infiltrate may be seen. Histologically, there appear cords of cancer cells, as well as single cells infiltrating the stroma of breast cancer $[8,10,12]$. Microscopically, ductal carcinomas tend to form glandular structures, and the tumor cells show a variable degree of differentiation. Cytological characteristics of malignant cells are expressed in varying degrees [13]. Some of the tumors diagnosed as invasive ductal breast cancer of no special type (IDC-NST) show a low degree of anaplasia, while others show a high degree of anaplasia [11, 13]. Cancer cells can invade lymph vessels and blood vessels, as well as nerve trunks. About $80 \%$ of ductal/NST cancers show estrogen receptor (ER) positivity and about $25 \%$ are HER2-positive [7, 14].

The aim of this study was to evaluate the relationship between the histological grade, tumor size, and lymph node status in a group of 691 patients with IDC-NST.
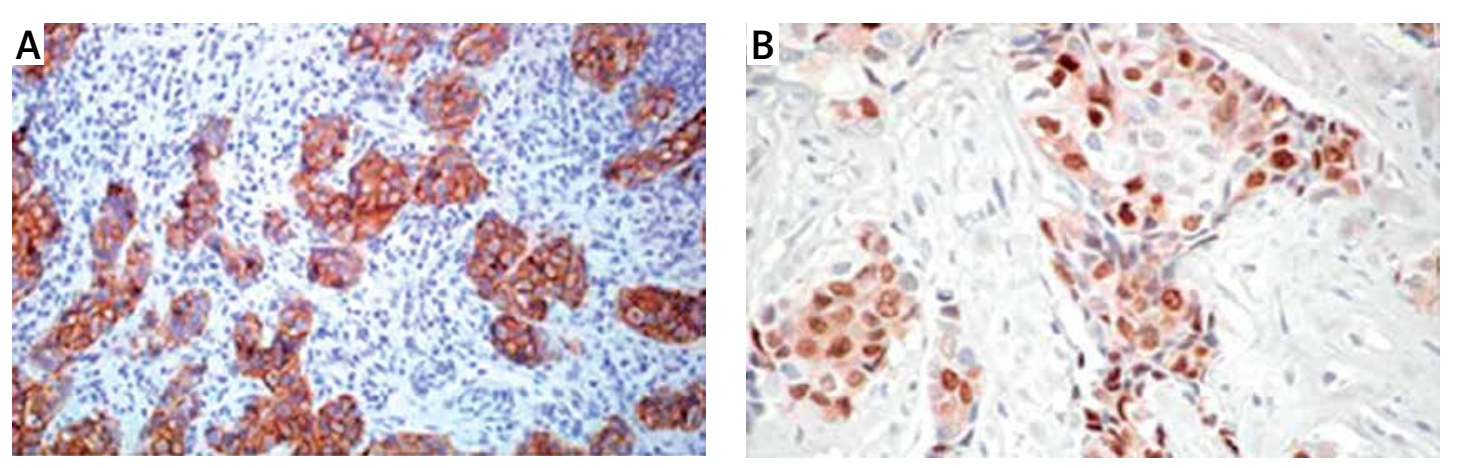

Figure 1. Immunohistochemical image of invasive ductal carcinoma of no special type of the breast (IDC-NST): A - Strong, circumferential staining in $>30 \%$ of invasive ductal carcinoma of no special type cells was scored as 3+, interpretation - positive for HER2 amplification, original magnification, 20x; B - Showing positive nuclear staining of progesterone receptor in IDC-NST of the breast, original magnification, 20x

\section{Material and methods}

The material consisted of histological preparations derived from 691 patients treated for IDCNST. The material for the study came from biopsy, excisional biopsy, and modified radical mastectomy. Histological and immunohistochemical studies were performed at the Department of Pathology, Military Medical Institute in Warsaw. Samples of tumors were fixed in $10 \%$ phosphate buffered formalin. Paraffin blocks were cut into sections with a thickness of $4 \mu \mathrm{m}$ each. The obtained sections were stained with different methods for diagnostic purposes. Preparations stained with hematoxylin and eosin were used to identify tumor type (WHO classification), tumor grade including tubule formation, intensity of division, and degree of mitotic index of neoplastic cells as the mean number of mitoses in neoplastic cells counted in 10 fields of vision at an objective magnification of $400 \times$ (surface field $0.17 \mathrm{~mm}^{2}$ ). Routinely, patients with IDC-NST had a basic immunohistochemical profile assessed, i.e. expression of estrogen receptor (ER), HER2 (Figure $1 \mathrm{~A}$ ) and progesterone receptor (PgR) (Figure $1 \mathrm{~B}$ ).

\section{Statistical analysis}

All statistical analyses were performed with SPSS software version 12.0 for Windows. If the expected size was less than 5 , the chi-square $\left(\chi^{2}\right)$ test was used with Yates' correction.

The correlations between variables were assessed using Spearman's rank or Cramer's V coefficient. The Spearman rank-order correlation is the nonparametric version of the Pearson product-moment correlation. Its measures the strength of association between two ranked variables. To find the relation between qualitative (nominal) variables which cannot be ranked, Cramer's V coefficient was calculated.

To determine the relationship between the degree of histological malignancy $(G)$, lymph node

\author{
(1)
}

Arch Med Sci 6, October / 2017 
metastasis ( $\mathrm{pN}$ ) and tumor size ( $\mathrm{pT})$, lymph node metastasis $(\mathrm{pN})$ and the degree of histological malignancy $(\mathrm{G})$, and tumor size ( $\mathrm{pT}$ ) and metastatic lymph nodes (pN) in IDC-NST, the value of Cramer's $V$ coefficient was determined (a value of 0 indicated no relationship between variables). In order to investigate the correlation between the degree of histological malignancy $(\mathrm{G})$, lymph node metastasis $(\mathrm{pN})$ and tumor size ( $\mathrm{pT})$, lymph node metastasis $(\mathrm{pN})$ and the degree of histological malignancy $(\mathrm{G})$, and tumor size (pT) and metastasis to the lymph nodes $(\mathrm{pN})$ in patients with IDC-NST, Spearman's rho coefficient was determined. The results were considered as statistically significant if the $p$ value was $\leq 0.05$.

\section{Results}

Pathological examination of tumors obtained from 1122 patients suffering from breast cancer was carried out. The age of the patients ranged from 30 to over 81 years, with a mean age of 60.47 \pm 5.07 years. Patients were divided into seven age groups: under 30 years; from 31 to 40 years; from 41 to 50 years; from 51 to 60 years; from 61 to 70 years; from 71 to 80 years; and over 81 years (Figure 2). Among 1122 cases of breast cancer, there were 118 (10.52\%) invasive lobular carcinomas (ILC), 691 (61.59\%) IDC-NST, 29 (2.58\%) ductal carcinomas in situ (DCIS), 3 (0.27\%) lobular carcinomas in situ (LCIS), and 281 (25.04\%) cases of other breast cancers (Figure 3). Regarding the histological grade of malignancy, the largest group of IDC-NST comprised grade 2 (53.96\%) and grade 3 (28.98\%) (G2 and G3) tumors (Table I, Figure 4). During the analysis of the pre-operative

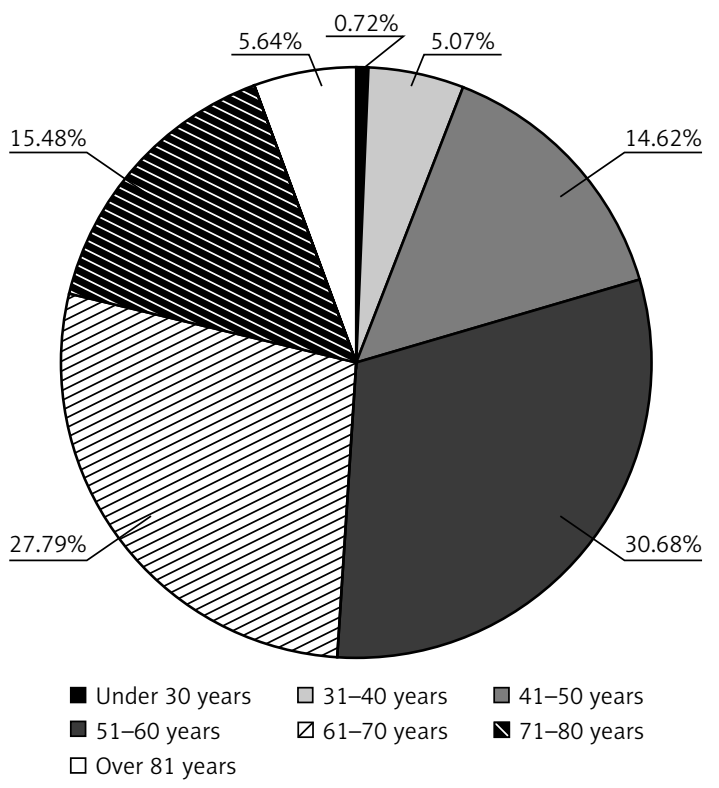

Figure 2. Age distribution of patients with IDC-NST staging of the studied cancers, it was found that the largest group of IDC-NST tumors consisted of those assessed as T1c (34.59\%) and T2 (35.31\%) (T1c - larger than $1 \mathrm{~cm}$, up to $2 \mathrm{~cm}$ in diameter; T2 - larger than $2 \mathrm{~cm}$ but not larger than $5 \mathrm{~cm}$ in diameter). None of the patients had tumor size of type T4a or T1d. Moreover, the size of the tumor of type T1a, T3, and T4b was found in fewer than $2 \%$ of patients with IDC-NST (Table I). The lymph node status was also assessed during the study. It was noted that in all investigated IDCNST cases, women without metastasis to regional lymph nodes constituted the largest group (pNo) (45.44\%). Furthermore, N1 and Nx was found in $14.47 \%$ and $2.89 \%$, respectively. In less than $0.5 \%$ of the study group, the N1B and N4 lymph node status was found (Table I). In $60.64 \%$ of patients, no distant organ metastases (M0) were identified, and in $39.36 \%$ those could not be assessed $(M x)$. Based on the analysis of the results of the relationship between histological grade $(\mathrm{G})$ and lymph node status ( $\mathrm{pN}$ ) in NST-IDC patients, and assuming the significance level of $p=0.05$ in the study, statistically significant correlations were found for N0, N1A, N1B, N3, N4 and G1-G3 (N0/ G1-G3 $p=0.0103 ; \mathrm{N} 1 \mathrm{~A} / \mathrm{G} 1-\mathrm{G} 3 p=0.0498$; N1B/ $\mathrm{G} 1-\mathrm{G} 3 p<0.001 ; \mathrm{N} 3 / \mathrm{G} 1-\mathrm{G} 3 p=0.0027 ; \mathrm{N} 4 / \mathrm{G} 1-\mathrm{G} 3$ $p<0.001$ ) (Table II).

The obtained results allowed us to evaluate the relationship between tumor size and lymph node metastasis in IDC-NST. We found a correlation between the size of T1-T4 tumor and lymph node status assessed as N0, N1B, N2, N2A, N4, and Nx (N0/T1-T4 $p=0.00295 ; \mathrm{N} 1 \mathrm{~B} / \mathrm{T} 1-\mathrm{T} 4 p<0.001$;

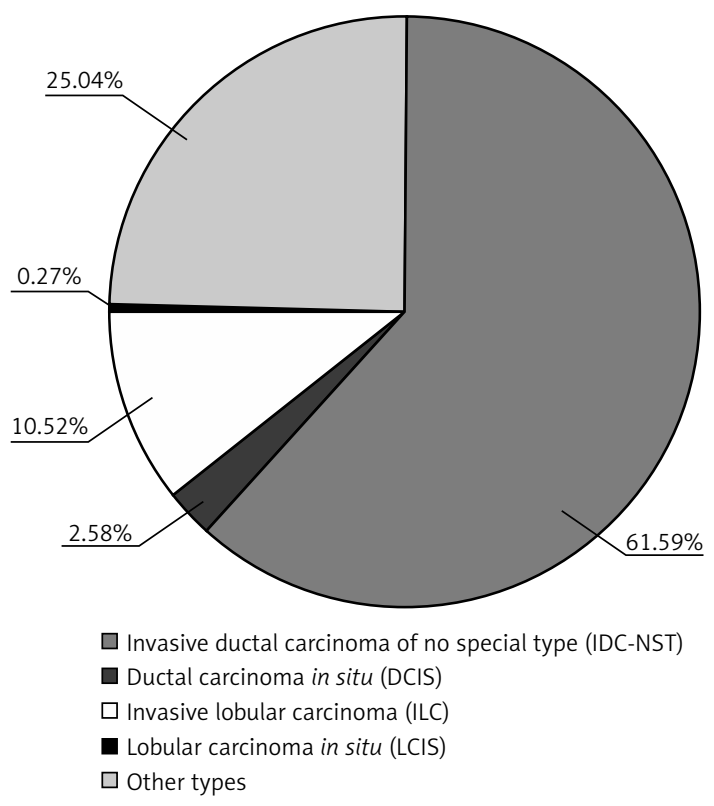

Figure 3. Histological types in the group of 1122 patients with breast cancer 
$\mathrm{N} 2 / \mathrm{T} 1-\mathrm{T} 4 p<0.001 ; \mathrm{N} 2 \mathrm{~A} / \mathrm{T} 1-\mathrm{T} 2 p<0.001 ; \mathrm{N} 4 / \mathrm{T} 1-\mathrm{T} 4$ $p<0.001 ; \mathrm{Nx} / \mathrm{T} 1-\mathrm{T} 4 \mathrm{p}=0.0447$ ) (Table II).

The analysis of the relationship between tumor size and lymph node metastasis allowed us to evaluate the clinical severity of the disease in patients with IDC-NST. Tumor, node, and metastasis (TNM) staging showed that the most common group was pT1N0 (39.74\%) (clinical stage I) followed by pT2NO (clinical stage IIA) (25.26\%), pT1N1 (clinical stage IIA) (14.90\%), and pT2N1 (clinical stage IIB) (12.52\%) (Table III). Based on the analysis of the test results, we also examined the relationship between tumor size (pT), and the degree of histological malignancy $(G)$. Our study demonstrated a statistically significant relationship between histological grade $\mathrm{G} 1$, $\mathrm{G} 1 / 2$, and $\mathrm{G} 2 / 3$, and tumor size T1-T4 (G1/T1$\mathrm{T} 4 p<0.001 ; \mathrm{G} 1 / 2 / \mathrm{T} 1-\mathrm{T} 4 p<0.001 ; \mathrm{G} 2 / 3 / \mathrm{T} 1-\mathrm{T} 4$ $p<0.0267$ ) (Table IV). We also examined the relationship between histological grade of the tumor, its size, and the presence of metastases to distant organs (Tables V, VI), but no statistically significant differences between the studied variables were found.

\section{Discussion}

Invasive ductal carcinoma of no special type is the most common form of microscopic breast cancer. According to most authors, it represents approximately $80 \%$ of all malignant breast tumors. In the literature it is assumed that the clinical picture of IDC-NST differs from that of clinical invasive lobular breast carcinoma (ILC) [11]. The main differences relate to patient age, tumor size at diagnosis, multifocal growth rate of cancer, and incidence of cancer in the other breast. Other important diagnostic markers are the degree of histological grade, lymph node status, and presence of distant metastases.

Our study was carried out in a group of 691 patients with ICD-NST. That group was isolated from 1122 patients with breast cancer. In our study, a detailed analysis of basic histopathological features of IDC-NST, such as the histological grade, primary tumor size, lymph node status, presence of distant metastases, and the clinical severity of the disease was carried out.

In our study the ages of the patients ranged from 30 to over 81 years, with a mean age of 60.47 years. Patients were divided into seven age groups: under 30 years; from 31 to 40 years; from 41 to 50 years; from 51 to 60 years; from 61 to 70 years; from 71 to 80 years, and over 81 years.

The data showed that the mean age of patients with IDC-NST is lower than the mean age of patients with ILC $[15,16]$. According to the results of Silverstein et al., the mean age of patients with IDC-NST was 52.5, according to Tubiana-Hulin et al.
Table I. Clinicopathologic characteristics of IDC-NST

\begin{tabular}{|c|c|c|}
\hline \multirow[t]{2}{*}{ Parameter } & \multicolumn{2}{|c|}{$\begin{array}{l}\text { Number of patients with } \\
\text { IDC-NST (total }=691 \text { ) }\end{array}$} \\
\hline & $N$ & $\%$ \\
\hline Age $($ mean $\pm S D)$ & \multicolumn{2}{|c|}{$60.47 \pm 5.07$} \\
\hline \multicolumn{3}{|l|}{ Tumor stage $(p T)$ : } \\
\hline $\mathrm{T} 1$ & 2 & 0.29 \\
\hline$T 1 a$ & 13 & 1.88 \\
\hline $\mathrm{T} 1 \mathrm{~b}$ & 55 & 7.96 \\
\hline T1c & 239 & 34.59 \\
\hline T1d & 0 & 0.00 \\
\hline $\mathrm{T} 2$ & 244 & 35.31 \\
\hline T3 & 9 & 1.30 \\
\hline T4 & 32 & 4.63 \\
\hline $\mathrm{T} 4 \mathrm{a}$ & 0 & 0.00 \\
\hline $\mathrm{T} 4 \mathrm{~b}$ & 6 & 0.87 \\
\hline Unknown & 91 & 13.17 \\
\hline \multicolumn{3}{|l|}{ Nodal stage $(\mathrm{pN})$ : } \\
\hline No & 314 & 45.44 \\
\hline $\mathrm{N} 1$ & 100 & 14.47 \\
\hline N1A & 41 & 5.93 \\
\hline N1B & 3 & 0.43 \\
\hline $\mathrm{N} 2$ & 34 & 4.92 \\
\hline $\mathrm{N} 2 \mathrm{~A}$ & 25 & 3.62 \\
\hline $\mathrm{N} 2 \mathrm{~B}$ & 4 & 0.58 \\
\hline N3 & 15 & 2.17 \\
\hline N3A & 17 & 2.46 \\
\hline N4 & 3 & 0.43 \\
\hline $\mathrm{Nx}$ & 20 & 2.89 \\
\hline Unknown & 115 & 16.64 \\
\hline \multicolumn{3}{|c|}{ Tumor grade (G1-3): } \\
\hline G1 & 52 & 7.48 \\
\hline G2 & 375 & 53.96 \\
\hline$\overline{\mathrm{G} 3}$ & 200 & 28.98 \\
\hline $\mathrm{G} 1 / 2$ & 2 & 0.29 \\
\hline $\mathrm{G} 2 / 3$ & 8 & 1.15 \\
\hline Unknown & 54 & 7.81 \\
\hline \multicolumn{3}{|l|}{ Metastases (pM): } \\
\hline$M x$ & 272 & 39.36 \\
\hline MO & 419 & 60.64 \\
\hline
\end{tabular}



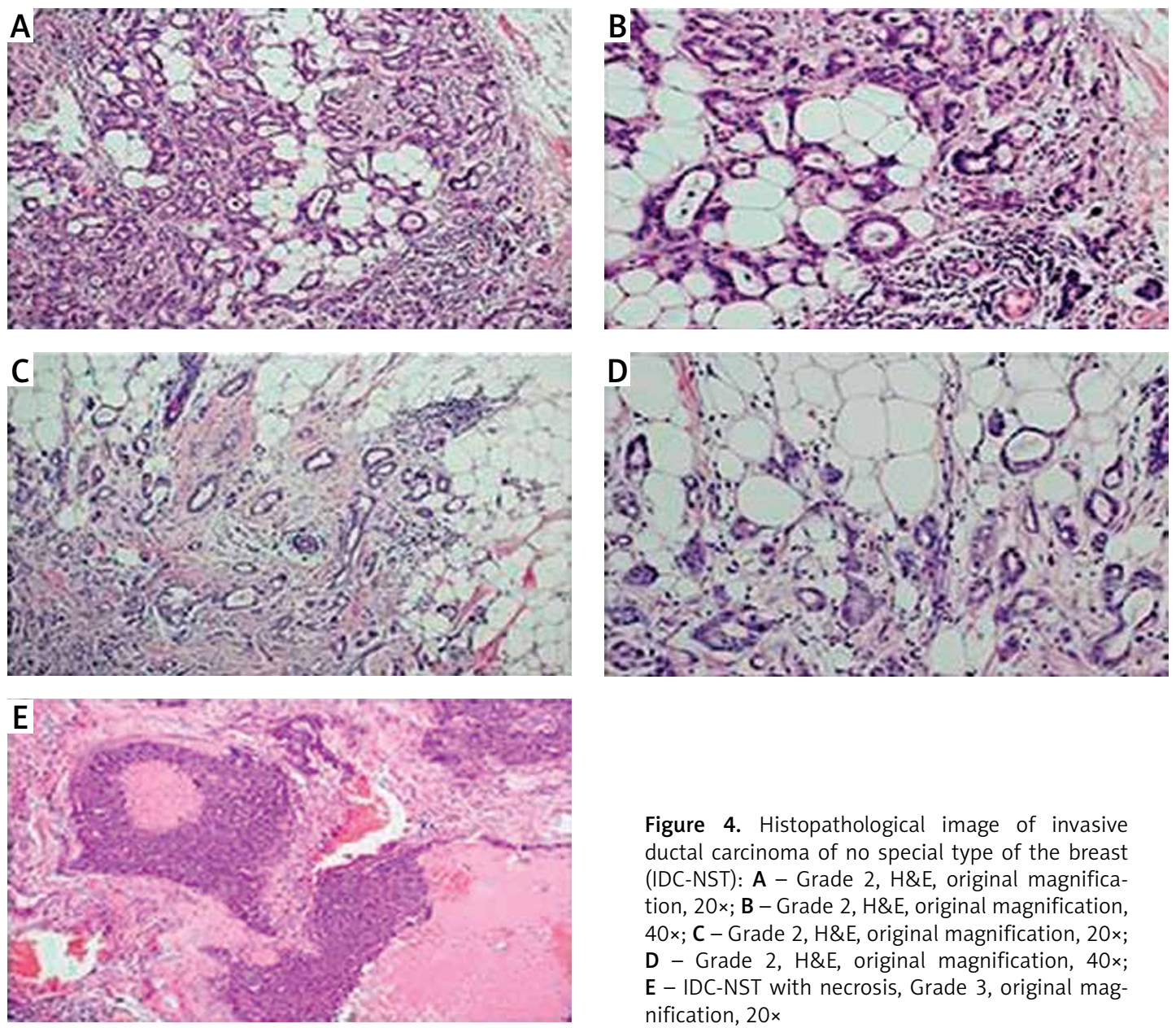

Figure 4. Histopathological image of invasive ductal carcinoma of no special type of the breast (IDC-NST): A - Grade 2, H\&E, original magnification, 20x; B - Grade 2, H\&E, original magnification, 40x; C - Grade 2, H\&E, original magnification, 20x D - Grade 2, H\&E, original magnification, 40x; E - IDC-NST with necrosis, Grade 3, original magnification, 20x

Table II. Relationship between degree of malignancy (G1-G3), tumor size and lymph node status (pN) in IDC-NST

\begin{tabular}{|c|c|c|c|c|c|c|c|c|c|c|c|c|c|c|c|c|}
\hline Tumor & G1 & $\mathrm{G} 2$ & G3 & $\mathrm{G} 1 / 2$ & $\mathrm{G} 2 / 3$ & $P$-value & $\mathrm{T} 1$ & T1a & T1b & T1c & T1d & $\mathrm{T} 2$ & T3 & T4 & $\mathrm{T} 4 \mathrm{~b}$ & $P$-value \\
\hline \multicolumn{17}{|c|}{ Invasive ductal carcinoma of no special type (IDC-NST): } \\
\hline NO & 32 & 184 & 81 & 1 & 2 & $0.0103^{*}$ & 1 & 7 & 33 & 143 & 0 & 117 & 1 & 9 & 0 & $0.00295^{*}$ \\
\hline N1 & 3 & 47 & 38 & 1 & 1 & 0.1201 & 1 & 1 & 6 & 33 & 0 & 42 & 1 & 4 & 1 & 0.9211 \\
\hline $\mathrm{N} 1 \mathrm{~A}$ & 8 & 22 & 7 & 0 & 1 & $0.0498^{*}$ & 0 & 0 & 5 & 18 & 0 & 16 & 0 & 1 & 0 & 0.9832 \\
\hline N1B & 0 & 2 & 1 & 0 & 0 & $<0.001^{*}$ & 0 & 0 & 0 & 5 & 0 & 0 & 0 & 0 & 0 & $<0.001^{*}$ \\
\hline N2 & 1 & 17 & 17 & 0 & 0 & 0.2193 & 0 & 0 & 0 & 10 & 0 & 13 & 2 & 8 & 0 & $<0.001^{*}$ \\
\hline $\mathrm{N} 2 \mathrm{~A}$ & 2 & 12 & 8 & 0 & 1 & 0.5508 & 0 & 0 & 1 & 3 & 0 & 13 & 1 & 3 & 4 & $<0.001^{*}$ \\
\hline N3 & 1 & 2 & 9 & 0 & 0 & $0.0027^{\star}$ & 0 & 0 & 1 & 4 & 0 & 7 & 2 & 1 & 0 & 0.03123 \\
\hline N3A & 0 & 10 & 6 & 0 & 1 & 0.2921 & 0 & 0 & 0 & 4 & 0 & 8 & 1 & 2 & 1 & 0.432 \\
\hline N4 & 0 & 2 & 1 & 0 & 0 & $<0.001^{*}$ & 0 & 0 & 0 & 1 & 0 & 1 & 0 & 1 & 0 & $<0.001^{*}$ \\
\hline Nx & 5 & 76 & 29 & 0 & 1 & 0.3318 & 0 & 2 & 10 & 12 & 0 & 11 & 0 & 1 & 0 & $0.0447^{\star}$ \\
\hline
\end{tabular}

*Statistically significant results $(p \leq 0.05)$.

it was 49, and according to Sastre-Garau et al. it was 56 [16-19]. In studies by Mersin et al., the mean age of patients with IDC-NST was 42 years [13]. According to the WHO, the mean age of patients with IDC-NST is from 1 to 3 years lower than in patients with ILC [20]. In our study, the mean age of patients with IDC-NST was 60.47 years.

In the study by Yeatman et al., $11.8 \%$ of patients with IDC were less than 39 years old [21], whereas in our study, women diagnosed with IDC- 
NST less than 39 years old accounted for $5.79 \%$. It should be noted, however, that for most of the authors, the differences in the mean age of patients with IDC are small and statistically insignificant [20-22].

Most of the data in the literature confirm that at the time of cancer diagnosis, the primary tumor in patients with IDC-NST is on average smaller in size than the primary tumor in patients with ILC [16-18, 21]. In studies by Yeatman et al., the average size of the primary tumor in patients with IDC-NST was $2.2 \mathrm{~cm}$, and in $4.5 \%$ of patients the tumor was classified as T3 [21]; in the study by Molland et al., in only $3 \%$ of patients with IDC the primary tumor was classified as T3 [23]. In the studies by Silverstein et al., the average size of the primary tumor in the group of patients with IDC was $2.3 \mathrm{~cm}$ [17]. In the material of Tubiana-Hulin et al. including 742 patients with IDC, $21.4 \%$ had a primary tumor of T3 stage [16]. In our study, the largest group among IDC-NST tumors consisted of those assessed as T1C (34.59\%) and T2 (35.31\%), and patients with IDC-NST classified as T3 and T4 accounted for $1.3 \%$ and $0.87 \%$, respectively (Table I).

In the microscopic evaluation of IDC-NST the following features are very important: the degree of cancer differentiation, invasion of the vascular space by cancer cells, and the status of regional lymph nodes. The quoted authors agreed that in
Table III. Clinical classification of IDC-NST

\begin{tabular}{|lccc|}
\hline $\begin{array}{l}\text { Tumor size } \\
\text { staging }\end{array}$ & $\begin{array}{c}\text { Node } \\
\text { involvement } \\
\text { staging }\end{array}$ & $\begin{array}{c}\text { Clinical } \\
\text { stage }\end{array}$ & Percent \\
\hline T1 $^{*}$ & NO & I & 39.74 \\
\hline T1 $^{*}$ & N1 & IIA & 14.90 \\
\hline T2 & N0 & IIA & 25.26 \\
\hline T2 & N1 & IIB & 12.52 \\
\hline T3 & NO & IIB & 0.21 \\
\hline T3 & N1* & IIIA & 0.21 \\
\hline T3 & N2 & IIIA & 0.65 \\
\hline T4 & N0-N2* & IIIB & 6.48 \\
\hline
\end{tabular}

*T1 includes T1a, T1b, T1c, T1d; T4 includes T4a, T4b; N1 includes N1A, N1B; N2 includes N2A.

IDC-NST, the percentage of patients with highly and moderately differentiated carcinoma $(\mathrm{G} 1, \mathrm{G} 2)$ is lower than in ILC $[13,16,18,23-27]$. According to the results obtained by Mersin et al., patients with highly and moderately differentiated cancer accounted for $46.6 \%$ of patients with IDC [13].

In the studies by Sastre-Garau et al., well-differentiated carcinoma was found in $22 \%$ of patients with IDC and poorly-differentiated cancer in $15 \%$ [18]. In the group of patients studied by

Table IV. Relationship between tumor size (pT) and degree of malignancy (G1-G3) in IDC-NST

\begin{tabular}{|c|c|c|c|c|c|c|c|c|c|c|}
\hline Tumor & T1 & T1a & $\mathrm{T} 1 \mathrm{~b}$ & T1c & T1d & $\mathrm{T} 2$ & T3 & T4 & $\mathrm{T} 4 \mathrm{~b}$ & $P$-value \\
\hline \multicolumn{11}{|c|}{ Invasive ductal carcinoma of no special type (IDC-NST): } \\
\hline G1 & 0 & 3 & 13 & 26 & 0 & 9 & 0 & 0 & 1 & $<0.001^{*}$ \\
\hline G2 & 1 & 2 & 25 & 137 & 0 & 127 & 5 & 15 & 3 & 0.6333 \\
\hline G3 & 1 & 2 & 11 & 59 & 0 & 88 & 4 & 14 & 1 & 0.1129 \\
\hline $\mathrm{G} 1 / 2$ & 0 & 0 & 0 & 1 & 0 & 1 & 0 & 0 & 0 & $<0.001^{\star}$ \\
\hline $\mathrm{G} 2 / 3$ & 0 & 1 & 1 & 1 & 0 & 3 & 0 & 1 & 0 & $0.0267^{*}$ \\
\hline
\end{tabular}

*Statistically significant results $(p \leq 0.05)$.

Table V. Relationship between degree of malignancy (G1-G3) and metastases to distant organs (pM) in IDC-NST

\begin{tabular}{|lcccccc|}
\hline Tumor & G1 & G2 & G3 & G1/2 & G2/3 & $P$-value \\
\hline Invasive ductal carcinoma of no special type (IDC-NST): & & & & \\
\hline M0 & 23 & 165 & 108 & 2 & 3 & 0.2993 \\
\hline Mx & 29 & 153 & 72 & 0 & 4 & 0.2993 \\
\hline
\end{tabular}

Table VI. Relationship between tumor size (pT) and metastases to distant organs in IDC-NST

\begin{tabular}{|ccccccccccc|}
\hline Tumor & T1 & T1a & T1b & T1c & T1d & T2 & T3 & T4 & T4b & $P$-value \\
\hline \begin{tabular}{l} 
Invasive ductal carcinom \\
\hline M0
\end{tabular} & 0 & 3 & 25 & 95 & 0 & 128 & 4 & 14 & 1 & 0.43587 \\
\hline Mx & 2 & 6 & 29 & 138 & 0 & 116 & 4 & 20 & 2 & 0.43587 \\
\hline
\end{tabular}


Tubiana-Hulin et al., those with highly and moderately differentiated IDC accounted for $45.4 \%$ [16]. In the studies by Mathieu et al., $63 \%$ of patients with IDC had highly or moderately differentiated carcinoma [26]. In the studies of Cristofanilli et al., poorly differentiated cancer was found in $56 \%$ of patients with IDC [25].

In our study, $7.48 \%$ of patients had well-differentiated IDC-NST, $53.96 \%$ had moderately differentiated IDC-NST, and $28.98 \%$ had poorly differentiated IDC-NST. In patients with IDC, lymph node metastasis was found more frequently than in patients with ILC $[16-19,28]$. In the studies by Silverstein et al., metastases to axillary lymph nodes were found in $37 \%$ of patients with IDC [17], while in our study, lymph node metastases were found in $34.58 \%$ of patients with IDC-NST. Tubiana-Hulin et al. in their study found no metastases to lymph nodes in $43.3 \%$ of patients with IDC [16]. In our study, no metastases were found in $45.44 \%$ of patients with IDC-NST. In order to investigate the correlation between clinical features of IDC-NST, Spearman's rho coefficient was determined. The obtained results indicated a correlation between the degree of histological grade (G1 and $\mathrm{G} 2$ ) and lymph node metastasis [G1/pN (-0.70743); G2/pN $(-0.72177)]$. Moreover, a correlation between the size of the tumor pT1c and lymph node metastasis $[\mathrm{T} 1 \mathrm{c} / \mathrm{pN}(-0.86715)]$ was found.

In conclusion, IDC-NST constitutes more than a half of all diagnosed histological types of breast cancer in women. The most common among patients with IDC-NST are cases showing grade 2 (G2). Among IDC-NST cases, the most common are T1C and T2. The largest group of patients with IDCNST consisted of those in whom the disease severity was as assessed as I and IIA. A relationship was found between IDC-NST of histological grades 1 and 2 and presence of metastasis to lymph nodes. In more than a half of the patients with IDC-NST, there were no metastases to distant organs.

\section{Acknowledgments}

I sincerely thank Dr J. Patera for help in implementing the project.

\section{Conflict of interest}

The authors declare no conflict of interest.

\section{References}

1. Ellis IO, Galea M, Broughton N, et al. Pathological prognostic factor in breast cancer. II. Histological type. Relationship with survival in a large study with long-term follow-up. Histopathology 1992; 20: 479-89.

2. Weigelt B, Geyer FC, Reis-Filho JS. Histological types of breast cancer: how special are they? Mol Oncol 2010; 4: 192-208.
3. Rakha EA, El-Sayed ME, Lee AH, et al. Prognostic significance of Nottingham histologic grade in invasive breast carcinoma. J Clin Oncol 2008; 26: 3153-8.

4. Yerushalmi R, Hayes MM, Gelmon KA. Breast carcinoma - rare types: review of the literature. Ann Oncol 2009; 20: 1763-70.

5. Junjeong C, Woo-Hee J, Ja Seung K. Clinicopathologic features of molecular subtypes of triple negative breast cancer based on immunohistochemical markers. Histol Histopathol 2012; 27: 1481-93.

6. Elston CW, Ellis IO. Pathological prognostic factor in breast cancer. I. The value of histological grade in breast cancer: experience from a large study with long-term follow-up. Histopatology 1991; 19: 403-10.

7. Badowska-Kozakiewicz AM, Sobol M, Patera J, et al. Immunohistochemical evaluation of human epidermal growth factor receptor 2 and estrogen and progesterone receptors in invasive breast cancer in women. Arch Med Sci 2013; 9: 466-71.

8. Rosen PP. Rosen's breast pathology. Lippincots-Raven Publishers, 1997.

9. Simpson P, Reis-Filho J, Gale T, et al. Molecular evolution of breast cancer. J Pathol 2005; 205: 248-54.

10. Weidner N, Cote R, Suster S, et al. Modern surgical pathology. Elsevier Science, 2003.

11. Turashvili G, Bouchal J, Ehrmann J, et al. Novel immunohistochemical markers for the differentiation of lobular and ductal invasive breast carcinoma. Biomed Pap Med Fac Univ Palacky Olomouc Czech Repub 2007; 151: 59-64.

12. Toikkanen S, Pylkkanen L, Joensuu H. Invasive lobular carcinoma of the breast has better short- and long-term survival than invasive ductal carcinoma. Br J Cancer 1997; 76: 1234-40.

13. Mersin H, Yildirim E, Gulben K, et al. In invasive lobular carcinoma different from invasive ductal carcinoma? Eur J Surg Oncol 2003; 29: 390-5.

14. Atik E, Guray M, Ozgur T, et al. Characterization of immunohistochemical markers in triple negative breast carcinomas. JBUON 2013; 18: 886-90.

15. Hussien M, Lioe TF, Finnegan J, et al. Surgical treatment for invasive lobular carcinoma of the breast. Breast 2003; 12: 23-35.

16. Tubiana-Hulin M, Stevens D, Lasry S, et al. Response to neoadjuvant chemotherapy in lobular and ductal breast carcinomas: a retrospective study on 860 patients from one institution. Ann Oncol 2006; 17: 1228-33.

17. Sliverstein MJ, Lewinsky BS, Waisman JR, et al. Infiltrating lobular carcinoma. Is it different from infiltrating duct carcinoma? Cancer 1994; 73: 1673-7.

18. Sastre-Garau X, Jouve M, Asselain B, et al. Infiltrating lobular carcinoma of the breast. Clinicopathologic analysis of 975 cases with reference to date on conservative therapy and metastatic patterns. Cancer 1996; 77: 113-20.

19. World Health Organization. Classification of tumors. Pathology and genetics of tumours of the breast and female genital organs. Tavassoli FA, Devilee P (eds). IARC Press, Lyon 2003; 23-5.

20. Winchester DJ, Chang HR, Graves TA, et al. A comparative analysis of lobular and ductal carcinoma of the breast: presentation, treatment, and outcomes. J Am Coll Surg 1998; 186: 416-22.

21. Yeatman TJ, Cantor AB, Smith TJ, et al. Tumor biology of infiltrating lobular carcinoma. Implications for management. Ann Surg 1995; 22: 549-61.

22. du Toit RS, Locker AP. Ellis IQ, et al. An evaluation of differences in prognosis, recurrence patterns and receptor 
status between invasive lobular and other invasive carcinomas of the breast. Eur J Surg Oncol 1991; 17: 251-7.

23. Molland JG, Donnellan M, Janu NC, et al. Infiltrating lobular carcinoma - a comparison of diagnosis, management and outcome with infiltrating duct carcinoma. Breast 2004; 13: 389-96.

24. Cocquyt VF, Blondeel PN, Depypere HT, et al. Different re sponse to preoperative chemotherapy for invasive lobular and invasive ductal breast carcinoma. EJSO 2003; 29: 361-7.

25. Cristofanilli M, Gonzalez-Angulo A, Sneige N, et al. In vasive lobular carcinoma classic type: response to primary chemotherapy and survival outcomes. J Clin Onco 2005; 23: 41-8.

26. Mathieu MC, Rouzier R, Llombart-Cussac A, et al. The poor responsiveness of infiltrating lobular breast carcinomas to neoadjuvant chemotherapy can be explained by their biological profile. Eur J Cancer 2004; 40: 342-51.

27. Sinha PS, Bendall S, Bates T. Does routine grading of invasive lobular cancer of the breast have the same prognostic significance as for ductal cancers? Eur J Surg Oncol 2000; 26: 733-7.

28. Toikkanen S, Pylkkanen L, Joensuu H. Invasive lobular carcinoma of the breast has better short and long-term survival than invasive ductal carcinoma. $\mathrm{Br} J$ Cancer 1997; 76: 1234-40. 